\title{
Factores que motivan la compra por impulso en el contexto de la compra en grupo en línea
}

Fecha de recepción: 16 de junio de 2019

Fecha de aprobación: 09 de diciembre de 2020

Fecha de publicación: 26 de abril de 2021

Cómo citar este artículo / To reference this article / Comment citer cet article / Para citar este artigo:

Rodríguez-Orejuela, A.; Peña-García, N.; Casañas-Chavez, M. I. (2020). Factores que motivan la compra por impulso en el contexto de la compra en grupo en línea. Revista Escuela de Administración de Negocios, (89), 177-196.

DOI: https://doi.org/10.21158/01208160.n89.2020.2846

\section{Resumen}

Este estudio tiene como propósito entender los factores que promueven la compra por impulso en sitios web de compra en grupo, a través del enfoque de la teoría psicológica ambiental de estímulo-organismorespuesta (S-O-R). Se analizan los estímulos disponibilidad percibida del producto y atractivo visual, además de la reacción cognitiva y la telepresencia como organismos; finalmente, como respuesta, la urgencia a comprar impulsivamente. Se realizó una investigación cuantitativa por medio de una entrevista personal con cuestionario estructurado a 403 consumidores de tiendas de compra agrupada en línea. Los datos fueron tratados con EQS 6.3, a partir de un análisis factorial confirmatorio y ecuaciones estructurales con el fin de contrastar las hipótesis. Los resultados indican, por un lado, que una percepción positiva del consumidor respecto a la disponibilidad del producto y el atractivo visual en el sitio de compra en grupo en línea influyen positivamente en la telepresencia, así como esta a su vez influye positivamente en la sensación de compra por impulso en línea del consumidor; por otro, se observa que la reacción cognitiva va en contra de la urgencia por comprar por impulso. Así las cosas, se puede establecer las tiendas pueden gestionar la urgencia para comprar impulsivamente cuando se requiere realizar una actividad promocional; pero no puede gestionarse como una actividad promocional regular en una empresa que espere durar en el mercado.

Palabras clave: compra en línea; compra por internet; compra por impulso; compras en grupo en línea; teoría S-O-R; comportamiento del consumidor.

\footnotetext{
MBA - Universidad del Valle, Colombia. Doctor en Ciencias de Empresa - Universidad de Murcia, España. ORCID: https://orcid. org/0000-0003-2865-1748

2 Doctora en Marketing (Cum Laude) y Máster en Marketing e Investigación de Mercados - Universitat de València. Administradora de Empresas - Universidad del Valle. ORCID: https://orcid.org/0000-0002-6594-5940

3 Administradora de empresas y Magíster en Ciencias de la Organización - Universidad del Valle. ORCID: https://orcid.org/00000001-9461-5441
} 


\title{
Factors in urging impulse purchase in the context of online group buying
}

\begin{abstract}
The purpose of this study is to understand the factors that promote impulse purchase in group buying websites, through the theory of environmental psychological approach, Stimulus-Organism-Response (S-O-R). We analyzed the stimuli perceived availability of the product and visual appeal, besides the cognitive reaction and the telepresence as organisms, and the urge to buy impulsively as a response. A quantitative research was conducted by means of a personal interview with a structured questionnaire applied to 403 consumers of online group-buying stores. In order to test the hypotheses, the data were processed with EQS 6.3, based on a confirmatory factor analysis and structural equations. The results show, on the one hand, that a positive perception of the consumer regarding product availability and visual appeal in the online group buying website influences telepresence in a positive way, and in turn, this positively influences the consumer's online impulse purchase sensation; on the other hand, it shows that the cognitive reaction goes against the urge to buy impulsively. Thus, it can be established that stores can manage the urgency to buy impulsively when a promotional activity is required; but it cannot be managed as a regular promotional activity in a company that expects to last in the market.
\end{abstract}

Keywords: online shopping; internet shopping; impulse purchase; online group buying; S-O-R theory; consumer behavior.

\section{Fatores que motivam a compra por impulso no contexto da compra coletiva online}

\section{Resumo}

Este estudo tem como objetivo compreender os fatores que promovem a compra por impulso em sites de compras coletivas, por meio da abordagem da teoria psicológica ambiental de estímulo-organismo-resposta (S-O-R). Foram analisados os estímulos de disponibilidade percebida do produto e atratividade visual, além da reação cognitiva e a telepresença como organismos e finalmente, como resposta, a necessidade de comprar impulsivamente. Realizou-se uma pesquisa quantitativa através de uma entrevista pessoal com questionário estruturado a 403 consumidores de lojas de compras coletivas online. Os dados foram tratados com EQS 6.3, a partir de uma análise fatorial confirmatória e equações estruturais para contrastar as hipóteses. Os resultados indicaram, por um lado, que uma percepção positiva do consumidor quanto à disponibilidade do produto e o atrativo visual no site de compras coletivas online influenciam positivamente na telepresença, que por sua vez influencia positivamente a sensação de compra por impulso do consumidor online e, por outro lado, observou-se que a reação cognitiva vai no sentido contrário à necessidade de comprar por impulso. Assim, é possível estabelecer lojas que consigam administrar a necessidade de comprar impulsivamente quando uma ação promocional é necessária, mas não pode ser administrado como uma atividade promocional regular em uma empresa que espera permanecer no mercado.

Palavras-chave: compra online; compra por internet; compra por impulso; compras coletivas online; Teoria S-O-R; comportamento do consumidor. 


\section{Facteurs incitatifs d'achats impulsifs en ligne au travers d'achats groupés}

\section{Résumé}

Cette étude a pour but de comprendre les facteurs motivant les achats impulsifs sur des sites d'achats groupés au travers de la théorie psychologique environnementale stimulus-organisme-réponse (S-O-R). Nous analyserons les stimuli perçus en fonction de la disponibilité du produit, l'attractivité visuelle, la réaction cognitive, la présence virtuelle et finalement l'acte d'achat impulsif. Une étude quantitative sera réalisée au travers d'entretiens personnalisés et de questionnaires structurés auprès de 403 consommateurs participant à des achats groupés sur des sites en ligne. Les données seront traitées avec le logiciel EQS 6.3 au travers d'une analyse factorielle confirmatoire et d'équations structurelles pour la confirmation des hypothèses. Les résultats indiquent d'une part, qu'une perception positive du consommateur sur la disponibilité du produit et l'attrait visuel du site d'achat groupé influencent positivement la présence virtuelle, influençant à son tour positivement la sensation d'achat impulsif du consommateur en ligne. D'autre part, nous constatons que la réaction cognitive va à l'encontre de l'achat impulsif. Il est ainsi possible de créer des sites capables de gérer l'urgence d'achats impulsifs des consommateurs lorsqu'une activité promotionnelle est en cours, sans qu'il s'agisse d'une activité promotionnelle courante pour une entreprise qui souhaite pérenniser sa présence sur le marché.

Mots-clés: achats en ligne; achats impulsifs; achats de groupe en ligne; Théorie S-O-R; comportement du consommateur. 


\section{Introducción}

$\mathrm{I}$ nternet se ha convertido en una herramienta que no solo cambió la manera en la que nos comunicamos, también la manera en la que se realizan negocios por medio del canal de venta en línea (Klaus y Nguyen, 2013). Además, a medida que el comercio electrónico empezó a generalizarse, comenzaron a surgir nuevas exploraciones en la literatura científica. Por una parte, algunos estudios se concentraron en caracterizar el perfil del consumidor en línea, por ejemplo, al abordarlo como una persona de carácter innovador que realiza búsquedas de conveniencia, es decir, búsquedas que reduzcan su esfuerzo físico y permitan flexibilidad de tiempo para realizar transacciones (Lim y Cham, 2015). Por otra, otros estudios se centraron en entender las razones detrás del comercio electrónico y las maneras de uso comercial más eficaces, de manera que muestran cómo diversos estímulos de un ambiente en línea, derivados de estrategias de marketing, podrían influir en el comportamiento de compra del consumidor, lo que ocasiona, incluso, compras no planeadas (Gillenson y Sherrell, 2002).

Tanto en las compras tradicionales como en las que se realizan en línea las personas suelen tener una lista previa; no obstante, muchas veces terminan por hacer compras no planificadas o compras por impulso (Koufaris, Kambil y LaBarbera, 2001). Además, pese a que el ambiente del comercio electrónico se encuentra reducido a la visibilidad de una pantalla, ciertas condiciones en la compra pueden afectar el uso tanto previsto como real, así como los resultados de las compras — satisfacción, cantidad comprada y tiempo gastado en la tienda virtual - a través de la influencia en los estados emocionales y cognitivos del consumidor (Eroglu, Machleit y Davis, 2001).

Por otra parte, las compras en grupo, que han sido una modalidad de compra común en el comercio tradicional, gracias a internet han pasado al contexto virtual. Este nuevo concepto del comercio minorista -aplicado, sobre todo, en pequeñas y medianas empresas - busca ofrecer productos/servicios a bajo costo por un tiempo limitado, con el fin de aprovechar el poder de compra de los consumidores individuales y grupales ( $\mathrm{Liu} \mathrm{Li}$ y $\mathrm{Hu}, 2013$ ). Este modelo se desarrolló en el 2008 en Estados Unidos, con Groupon.com.co (Zhou, Xu y Liao, 2013); la plataforma cuenta con un total de 110 millones de personas en el mundo conectadas desde su smartphone, incluyendo Colombia (Iglesias, $01 \mathrm{de}$ abril de 2015). En vista de su éxito, ha sido replicado por otros competidores como Cuponatic.com y Quebuenacompra.com, entre otros.

En consecuencia, frente a las diversas ventajas de las compras en grupo en línea, esta modalidad presenta una razón importante para analizar la compra por impulso debido a que el límite de tiempo en las ofertas, posiblemente, atraerá compradores impulsivos a participar en las compras grupales (Liu et al., 2013). De modo que incentivar sentimientos positivos mientras se genera una distorsión del tiempo para el consumidor podría mantener su atención y, así, mayor tiempo de exposición a estímulos que luego, a partir de un procesamiento interno, conducirán a un comportamiento de compra por impulso (Xiang, Zheng, Lee y Zhao, 2016).

Las compras en grupo en línea se utilizan con el fin de atraer clientes que buscan productos o servicios que no son de primera necesidad. De igual modo, las decisiones de compra se toman, por lo general, durante el tiempo libre, lo cual podría ocasionar que muchas decisiones sobre servicios de ocio se tomen por impulso (Langviniene, Zemblyte y Sliziene, 2016). Además, el tiempo limitado de la oferta podría también ocasionar este comportamiento si se tiene en cuenta que la mayoría de compras por impulso se toman deprisa. A partir de lo anterior, este estudio pretende conocer y entender los antecedentes que determinan la compra por impulso en las compras en grupo en línea, desde la teoría psicológica ambiental de estímulo-organismo-respuesta o S-O-R, por sus siglas en inglés. 
A fin de alcanzar el objetivo de la investigación, el documento presenta una revisión de la literatura sobre las compras por impulso, la teoría S-O-R y las compras en grupo en línea en el segundo apartado. A continuación, se presenta la metodología propuesta con el fin de contrastar las hipótesis enunciadas en el modelo de la investigación. Posteriormente, se presentan los resultados obtenidos y su discusión. El documento finaliza con las conclusiones y las limitaciones de la investigación.

\section{Revisión de la literatura}

\subsection{Compras por impulso}

$\mathrm{I}^{\mathrm{n}}$ nicialmente, las investigaciones de compra por impulso se enfocaron en estudiar la compra y no al consumidor, es decir, la diferencia entre las compras previstas y las compras reales de este. De esta manera, la compra por impulso se definió como cualquier compra no planeada. Sin embargo, Applebaum (1951) y Piron (1991) sugirieron que estos impulsos podrían ser resultado de la exposición del consumidor a una serie de estímulos, y es entonces cuando el comportamiento del consumidor dejó de entenderse como una toma de decisiones a partir de aspectos racionales o cognitivos, y más bien por medio de decisiones que influyen otros factores vinculados con el proceso de compra (Piron, 1991).

Stern (1962) identifica cuatro tipos de compras por impulso: la compra pura, como aquel impulso emocional generado por la variedad y la novedad de productos; la compra planeada, cuando el comprador tiene pensado realizar unas compras específicas, sin embargo, posee la intención de realizar otras compras dependiendo de los precios especiales, las ofertas promocionales, los descuentos y otros factores; la compra sugerida, basada en las indicaciones del vendedor y en la que el comprador carece de conocimiento y experiencia previa del producto; por último, la compra por recordación, la cual se produce cuando el cliente recuerda una necesidad de compra al observar un anuncio o por actividades de promoción de productos.

Más adelante, Rook (1987) define la compra por impulso como una sensación repentina, poderosa y persistente; además, el deseo de compra por impulso es hedónicamente complejo y deriva -en gran medida - en un conflicto emocional del comprador (Sofi y Najar, 2018). Ahora bien, se debe tener en cuenta que los estados de ánimo - positivos o negativos-, pese a ser difusos y de corta duración, afectan el comportamiento del consumidor y pueden motivar o no un impulso de compra (Rook y Gardner, 1993). Del mismo modo, es importante reconocer que la toma de decisión del consumidor puede estar también determinada por juicios —desarrollo cognitivo - y comportamiento, es decir, en las compras por impulso existe una fuerte relación entre la cognición y el afecto (Sofi y Najar, 2018).

Madhavaram y Laverie (2004) añaden que la compra por impulso es el resultado de una reacción inmediata del comprador frente a unos estímulos externos que están cargados hedónicamente, lo cual implica un cambio en la intención de compra del consumidor derivado de la exposición a unos estímulos que no se limitan solo al producto, porque es el individuo quien experimenta el impulso.

En el comercio tradicional, Beatty y Ferrell (1998) indican que las compras por impulso están dominadas por el tiempo y el dinero disponible, así como por el disfrute en el proceso de compra y la tendencia a las compras por impulso. Entretanto, en el comercio electrónico las condiciones permiten al consumidor ahorrar tiempo y, de esta manera, deriva en las compras por impulso (Koufaris, Kambil y LaBarbera, 2001). 


\subsection{Teoría S-O-R}

Los estudios más utilizados en investigaciones sobre la compra por impulso en línea, de acuerdo con Xiao y Nicholson (2013), se apoyan en diversas teorías, tales como la teoría estímulo-respuesta y los conceptos de estímulos ambientales; las teorías económicas y los modelos de precios de promoción relacionados; las teorías socioculturales; los conceptos de procesamiento emocional y psicológico; los rasgos de personalidad; el autocontrol y las teorías de regulación; así como en el sistema dual de la teoría reflexiva.

La presente investigación se aborda desde la teoría S-O-R, porque a la luz de la literatura ha demostrado ser la más conveniente a fin de entender el comportamiento del consumidor (Chan, Cheung y Lee, 2016). La teoría S-O-R fue ajustada de la psicología ambiental, un movimiento que nació con el fin de estudiar el comportamiento humano y abordar factores psicológicos tales como actitudes, conocimientos y creencias ambientales, y la manera cómo estas variables se ven afectadas por la interacción individuo-medio ambiente (López, 2006).

La teoría S-O-R fue aplicada en investigaciones del área de marketing por los pioneros Mehrabian y Russell (1974), quienes intentaron evidenciar, bajo el enfoque de la tienda minorista, que los estímulos ambientales - estímulo - influyen en los estados emocionales del consumidor - organismo-, y que esto, a su vez, afecta el acercamiento o evitación de respuestas — respuesta-, es decir, el deseo o no de quedarse, mirar a su alrededor y explorar el medio ambiente (Jang y Namkung, 2009). En otras palabras, la gente reacciona a las influencias del medio ambiente, ya sea de manera positiva - comportamiento de acercamiento- o de manera negativa - conducta de evitación-, donde los estados emocionales sirven como mediadores o variables intervinientes para completar la secuencia del modelo S-O-R (Floh y Madlberger, 2013). Así mismo, el comportamiento de aproximación-evitación incluye tres aspectos importantes: el deseo de explorar el entorno, el deseo de interactuar en el medio ambiente y la satisfacción percibida en el entorno (Vieira, 2013).
El marco S-O-R intenta incluir diferentes características de un sitio en línea, como, por ejemplo, estímulos ambientales. También examina el papel de las reacciones afectivas y cognitivas del medio ambiente en línea sobre la probabilidad de experimentar un impulso de compra, así como estudiar el impulso en su calidad de estado de ánimo que resulta de la exposición de las señales ambientales de la tienda en línea (Parboteeah, Valacich, y Wells, 2009).

Específicamente en la respuesta del consumidor, en la revisión de la literatura, se encuentra que se puede dividir en dos etapas. La primera se refiere a la necesidad de comprar impulsivamente, es decir, el estado de deseo que experimenta el consumidor al exponerse a un producto/servicio en el entorno. La segunda es la compra por impulso real generada después de que el consumidor experimenta la necesidad de comprar de manera impulsiva (Zheng et al., 2019). Por tanto, el modelo presenta la necesidad de estudiar por separado la urgencia a comprar impulsivamente y la compra por impulso; la primera es la sensación de comprar por impulso, mientras la segunda es la respuesta a esa sensación. Estos sentimientos son más fuertes en el comercio electrónico en razón a la oferta de precios bajos o el encuentro con ofertas especiales (Bressolles, Durrieu y Giraud, 2007). El presente estudio se centra en la urgencia por comprar de forma impulsiva.

\subsection{Compras en grupo en línea}

De acuerdo con Kauffman y Wang (2002), los primeros sitios de compras en grupo en línea incursionaron en el mercado de subastas y se establecieron en la década de los noventa en mercados estadounidenses - Mercata.com y Acompany.comy europeos -CoShopper.com-, con el propósito de disminuir el precio del producto a medida que aumentaba el número de grupo de compradores; sin embargo, la mayoría de estos primeros sitios web cesaron sus operaciones por falta de compresión del modelo. 
Aun así, pese al fracaso de algunos sitios web de subastas, para Zhang et al. (2016) los consumidores siempre se han reunido con el fin de negociar descuentos por volumen de compra y, como resultado de los avances del comercio electrónico y las redes en línea, las compras en grupo resurgieron a través de plataformas en línea dedicadas a la venta de productos de diversos vendedores.

Los sitios de compras en grupo en línea han sido populares en los últimos diez años (Zhang et al., 2016) ya que, por un lado, permiten a empresas en periodo de crisis acudir a nuevos clientes y obtener beneficios publicitarios, sobre todo para aquellos que no gozan de un alto reconocimiento, porque un gran número de consumidores son informados de sus promociones (Erdogmus y Cicek, 2011). Por otro, para el consumidor brinda la sensación de pagar un precio final inferior al ofrecido por otros vendedores, lo que representa un ahorro y la sensación de sentir poder de negociación con el vendedor (Kauffman y Wang, 2001).

De igual manera, la popularidad de este modelo se presenta porque, de acuerdo con Zhang et al. (2016), primero, a diferencia de las compras tradicionales, las compras en grupo generan un entorno de red positivo a causa del intercambio de información que disminuye la carga cognitiva del consumidor y aumenta la demanda de un producto o servicio. Segundo, porque los vendedores tienen la facilidad de condicionar sus ventas a una cantidad mínima de compradores. En consecuencia, los vendedores ofrecen precios con descuentos y así generan cambios en las emociones de los consumidores que se traducen en satisfacción y actitud positiva a gastar más (Song, Chung Y Koo, 2015).
Las compras en grupo se pueden ver de dos maneras (Wang, Zhao y Li, 2013). La primera se trata de grupos de consumidores que buscan comprar productos, servicios o marcas específicas y actividades organizadas por las propias empresas con el propósito de promover sus productos a un grupo de compradores. En esta, el mecanismo de fijación de precios es dinámico, porque cambia a medida que aumenta el número de compradores, de modo que el mismo grupo realizará un esfuerzo para obtener más participantes y recibir un mejor precio (Erdogmus y Cicek, 2011); además, al finalizar la subasta, a cada comprador se le cobrará el mismo precio (Chen, Kauffman, Liu y Song, 2010).

La segunda, la cual será el objeto de la presente investigación, se da por medio de grupos intermediarios organizados por particulares $\mathrm{o}$ empresas con el fin de obtener un beneficio. Este tipo de modelo de negocio se denomina «ofertas del día» $\mathrm{u}$ «ofertas diarias» —en inglés conocido como today's deal o deal of the day-, las cuales son una forma de promoción de venta en la que un intermediario tiene una lista de suscriptores en su sitio web a través del correo electrónico y las redes sociales y ofrece acceso a esta lista a los vendedores a cambio de una comisión, lo que les permite promocionar sus ofertas con un alto descuento (Boon et al., 2015). Así, el vendedor arregla el precio de la compra en grupo y establece un tamaño de compra colectiva, bajo un precio fijo y no dinámico (Ni, Xu, Xu y Dong 2015), mientras el consumidor accede al producto/servicio mediante la compra de un cupón que se debe pagar por adelantado (Nakhata y Kuo, 2017).

\section{Modelo de investigación}

B asado en la revisión de la literatura, el estudio considera dos estímulos del ambiente en línea — disponibilidad percibida del producto y atractivo visual - que inciden en la reacción cognitiva — estado cognitivo-y afectiva —-telepresencia-, a la vez que modelan la urgencia a comprar impulsivamente (véase la Figura 1). 
Figura 1. Modelo de investigación

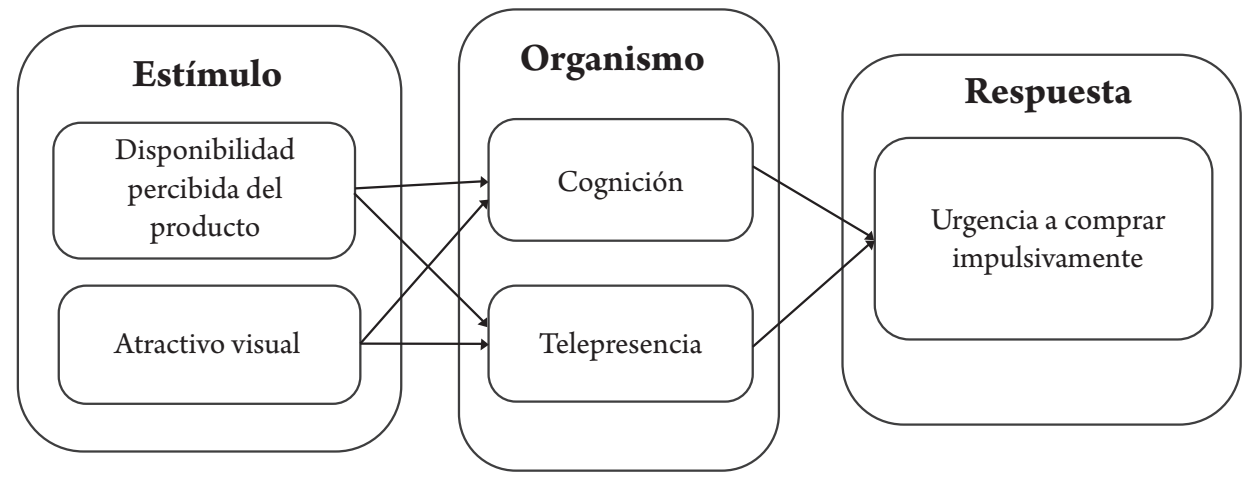

Fuente. Elaboración propia.

\subsection{Disponibilidad percibida del producto}

La disponibilidad percibida del producto, según Steinhart, Mazursky y Kamins (2013), es aquella que afecta los niveles de participación de los consumidores y la intención de compra; puede percibirse de manera negativa o positiva y está relacionada con las características prácticas del producto — es decir, la facilidad de obtenerlo- y menos relacionada con los sus beneficios principales. Positiva, al evocar en el producto aspectos de exclusividad — como productos populares o de limitada edición- que influirán en la participación de los consumidores; y negativa, cuando se presenta escasez del producto e influye en la viabilidad de realizarse una compra.

Por lo general, los consumidores se inclinan a buscar tiendas en las que encuentran y descubren otros productos que están actualmente disponibles. Dependiendo de cómo se muestren los productos, el consumidor puede percibir o no mayor variedad (Morales, Kahn, McAlister y Broniarczyk, 2005). Por tanto, la compra no depende solo del precio, la calidad y la disponibilidad, sino también del precio, la calidad y la disponibilidad de otros productos que el mismo cliente desee comprar (Flapper, GonzálezVelarde, Smith y Escobar-Saldívar 2010).

En el contexto de las compras en línea y, sobre todo, en las compras en grupo, según He y Oppewal (2018), los vendedores muestran en vivo el número de ventas y el nivel de existencia del producto, lo cual traduce al consumidor que un producto está disponible y es popular. Además, podrían señalar que tiene mayor calidad o valor $y$, de esta manera, influir en la decisión de compra del consumidor. En general, la disponibilidad del producto es una señal positiva porque, al estar disponible para la compra, el comprador lo encuentra como algo bueno, por tanto, la disponibilidad influye en el nivel de participación del consumidor (Steinhart et al., 2013). La variedad de productos también podría facilitar el proceso de búsqueda a los consumidores y encontrar productos que coincidan adecuadamente con sus gustos (Richards, Hamilton y Allender, 2016). Así mismo, la percepción positiva del consumidor sobre la disponibilidad de productos hará que evalué de forma positiva una compra por impulso no planificada y, a su vez, se reforzará el impulso a realizar la compra por impulso (Liu et al., 2013). Por tanto, se propone la siguiente hipótesis:

- H1: la disponibilidad percibida de producto del consumidor influye directa y positivamente en su estado cognitivo en una tienda de compra en grupo en línea.

En el contexto de las compras en grupo en línea, el atractivo de una mercancía se define por la percepción del tamaño y el atractivo del producto. De esta manera, las ofertas y los productos se verán relacionados con los intereses del comprador. Además, presentar ofertas interesantes puede crear 
emociones positivas (Verhagen y van Dolen, 2011). Una variedad de productos resultará en que los consumidores se sientan emocionados, satisfechos y contentos (Yoo, Park y MacInnis, 1998), ya que la preferencia de los consumidores a una amplia variedad de productos se ve impulsada por las experiencias que obtienen al revisarlos, debido a que la mayoría de productos son portadores de efectos positivos (Aydinli, Gu y Pham, 2017).

Vonkeman, Verhagen y van Dolen (2017) sugieren que la interactividad y la viveza del entorno en línea reducirá la percepción de distancia entre el consumidor y el producto o servicio, e influirá positivamente en compras por impulso; por tanto, la telepresencia media la relación entre la interactividad del sitio web y las impresiones de los usuarios, lo que resulta en actitudes más fuertes y más favorables. En consecuencia, se propone la siguiente hipótesis:

- H2: la disponibilidad percibida de producto del consumidor influye directa y positivamente en la telepresencia del consumidor en una tienda de compra en grupo en línea.

\subsection{Atractivo visual}

Skulmowski et al. (2016) apuntan que los juicios realizados por los usuarios varían de acuerdo con la confianza, el atractivo visual y la facilidad de uso, porque en una fracción de tiempo los consumidores desarrollan su primera sensación instintiva sobre un sitio web; además, posteriormente, deciden permanecer en la tienda o, por el contrario, navegar a otra. En consecuencia, el diseño de una tienda juega un papel incluso más importante en las compras en línea que en las compras tradicionales.

Soportados en lo anterior, el atractivo visual depende del diseño del sitio web, es decir, del grado en el que el cliente percibe la tienda como estéticamente agradable (Floh y Madlberger, 2013); también depende de la forma de presentar el producto para facilitar y estimular el comportamiento de compra de los consumidores (Oh, Fiorito y Hofacker, 2008). Por esto, un diseño web artístico será atractivo para los visitantes y, además, un diseño con valor estético será superiormente atractivo a otros con menos valor estético (Chang et al., 2014), y, cuanto mayor sea la calidad del ambiente de la tienda en línea, más comportamientos del consumidor se pueden desencadenar (Chen y Yao, 2018).

De acuerdo con Bitner (1992), el ambiente de una tienda puede provocar respuestas cognitivas, emocionales y fisiológicas que influirán en el comportamiento de aproximación o evitación de la tienda; este comportamiento de acercamiento se relaciona con el tiempo de permanencia en la tienda, el gasto de dinero y la cantidad de compra de productos.

La observación visual del sitio web permitirá al consumidor generar una reflexión cognitiva para tomar una decisión de preferencia hacia un producto (Lindgaard et al., 2011), de modo que la velocidad con la que el usuario tome le decisión depende de la impresión inmediata recibida del estímulo. Entonces, resulta evidente que el diseño de la página web influye en la imagen de la tienda en línea, un estado interno que, a su vez, afecta las expectativas de los consumidores como respuesta (Floh y Madlberger, 2013). Por todo esto, se propone la siguiente hipótesis:

- H3: el atractivo visual influye directa y positivamente en el estado cognitivo del consumidor en una tienda de compra en grupo en línea.

Madhavaram y Laverie (2004) argumentan que en un entorno en línea la compra por impulso será influenciada no solo por las características del producto, sino también por las características de la tienda en línea. Por tanto, una atmósfera que permita una experiencia agradable al consumidor influirá en sus emociones. Además, el diseño del sitio web también termina en resultados tales como la lealtad hacia la tienda, la cantidad de tiempo de permanencia en esta, el número de artículos comprados y la cantidad gastada (Mummalaneni, 2005). 
A diferencia de las tiendas físicas, en las tiendas en línea el consumidor puede percibir una ausencia de contacto humano y sociabilidad; por esta razón es importante que las señales del sitio web permitan una interacción humano-computadora, de tal manera que puedan percibirlo como algo natural (Hassanein y Head, 2005), ya que las personas tienden a tratar a la computadora como un actor social y no solo como un medio electrónico (Wang et al., 2007).

Como lo explican Ning-Shen y Khalifa (2012), en un entorno agradable los consumidores prestarán más atención, aumentando la probabilidad de seguir explorando el sitio web, y así lograr una urgencia de compra por impulso; mientras que en aquellos que experimentan un entorno desagradable abandonarán la página web. Con base en la discusión planteada, se propone la siguiente hipótesis:

- H4: el atractivo visual influye directa y positivamente en la telepresencia del consumidor en una tienda de compra en grupo en línea.

\subsection{Estado cognitivo}

De acuerdo con Coleyy Burgess (2003), la cognición se refiere al pensamiento, la comprensión y la interpretación de la información, es decir, el proceso mental producido cuando el consumidor interactúa con el estímulo de la tienda (Chan et al., 2016). Numerosos estudios han incluido el estado cognitivo en el proceso de compra por impulso como una variable dominante, sin embargo, los resultados no han sido claros, en razón a que excluyeron el afecto en el comportamiento del consumidor (Verhagen y van Dolen, 2011).

Otro estudio propuso el proceso cognitivo como una hipótesis relacionada negativamente con la compra por impulso (Lins et al., 2015), teniendo en cuenta que los compradores buscan comprar por materialismo - objeto deseado- y hedonismo atracción emocional-. Sin embargo, no obtuvieron un resultado que sustentara esta hipótesis y mucho menos que disminuyera el efecto del materialismo y el hedonismo.
Por lo anterior, el uso del proceso cognitivo en el modelo S-O-Rpresenta una discrepancia sobre si este debería ser parte o no del modelo, ya que la compra por impulso se caracteriza por ser una sensación de impulso repentina y presentar una disminución en el procesamiento de información y, por tanto, de reacciones cognitivas (Floh y Madlberger, 2013). Sin embargo, Ning-Shen y Khalifa (2012) manifiestan que, pese a que la compra por impulso se concibe, por lo general, como irracional, hedonista y no reflexiva, no está exenta de un proceso cognitivo, porque el consumidor podrá percibir el impulso como acorde a sus objetivos, recursos y situación, cuando la percepción de valor es coherente con su impulso, lo cual reforzará el impulso de compra.

De la misma manera, Verplanken y Herabadi (2001) exponen que la falta de planificación y de dominio de las emociones son características de las compras por impulso, pero las respuestas pueden derivarse tanto de procesos cualitativos - p. ej., considerar el costobeneficio de la compra- como de emociones, sin encontrarse exentas de un componente evaluativo, es decir, el comprador que planea sus compras y el que no podrían terminar en reacciones de impulso.

Por su parte, para Burroughs (1996) los consumidores realizan un procesamiento holístico de la información y evalúan decisiones de compra a gran velocidad, de tal forma que obtienen un significado simbólico que se puede comparar casi inmediatamente con la imagen que tiene el comprador; además, cuando se realiza una coincidencia, se realiza la compra. Según Chen y Yao (2018), los consumidores que se sienten positivos sobre la conveniencia de la compra por impulso tienen más probabilidades de mostrar conductas de compra por impulso. Apoyados en lo anterior, se propone la siguiente hipótesis:

- H5: el estado cognitivo del consumidor influye directa y positivamente en la urgencia por comprar de manera impulsiva en una tienda de compra en grupo en línea. 


\subsection{Telepresencia}

El concepto telepresencia se ha discutido en diversos campos, tales como el marketing, la informática y la psicología. Lo utilizó por primera vez Marvin Minsky en 1980, en referencia a sistemas de teleoperación para manipular los objetos físicos (Steuer, 1992; Lim y Ayyagari, 2018). En asuntos de compra en línea, la telepresencia se refiere a la percepción que tiene el comprador de estar presente en la ubicación virtual del vendedor, aunque físicamente el vendedor se encuentre alejado del comprador $(\mathrm{Ou}$, Pavlou y Davison, 2013).

Aunque la compra en línea es una de las áreas de crecimiento más rápido en el uso de internet, aún existen muchos consumidores con temor a realizar las compras en línea; entre sus razones, además de la inseguridad, se encuentran la incapacidad del consumidor de juzgar la calidad del producto puesto que no puede observar los productos en físico, razón por la cual muchas empresas crean hoy experiencias ambientales que generen un sentido convincente de telepresencia (Suh y Chang, 2006).

Las compras por impulso en línea han presentado un crecimiento considerable; sin embargo, muchos estudios se concentran en los fenómenos relacionados con la calidad del sitio web y la variedad de productos, de modo que prestan poca atención a la presentación de productos en entornos de comercio en línea que inducen a compras por impulso.

Según Steuer (1992), la telepresencia es la percepción del consumidor que simula una realidad sobre la tienda en línea. Esta percepción es el resultado de los estímulos virtuales que experimenten los navegadores y conforman un sentimiento subjetivo que solo percibe el consumidor (Gao et al., 2018). A su vez, la experiencia de telepresencia conducirá a una respuesta en el consumidor, por tanto, en caso de que el participante no sienta suficiente presencia, se puede sentir aburrido o no estimulado en la tienda (Heeter, 2003) y, finalmente, no conducir a una sensación de compra por impulso en línea, debido a que niveles altos de viveza en un sitio web pueden influir en niveles altos de telepresencia (Coyle y Thorson, 2001).

Por tanto, se propone la siguiente hipótesis:

- H6: la telepresencia influye directa y positivamente en la urgencia a comprar impulsivamente en una tienda de compra en grupo en línea.

\section{Metodología}

$\mathrm{C}$ on el fin de alcanzar el objetivo de la investigación, se propone realizar un estudio cuantitativo de corte transversal en compradores actuales o potenciales de los sitios de compra en línea agrupada. Para la recolección de información se diseñó un cuestionario estructurado, con el cual una compañía de investigación de mercados con panel de consumidores aplicó la encuesta a 450 consumidores en Colombia.

La información básica del cuestionario incluye seis preguntas de tipo nominal, ordinal y de intervalo que recopilan información relacionada con el género, la edad, el nivel de educación, la ocupación y las experiencias en compras en línea. A fin de evaluar el modelo de investigación, se desarrollaron siete preguntas medidas a través de escalas tipo Likert de 7 puntos de anclaje cuyos límites categorizan: $1=$ totalmente en desacuerdo y $7=$ totalmente de acuerdo.

Sobre la disponibilidad percibida del producto (DP) se utilizó el trabajo de Liu et al. (2013) y comprende tres ítems, al igual que para medir el atractivo visual $(\mathrm{AV})$, que también comprende tresítems. En relación con la medición de la variable telepresencia (TP) se tomó como referencia la escala de Ning-Shen y Khalifa (2012) y comprende cinco ítems. Respecto 
a la reacción cognitiva $(\mathrm{RC})$, se tomó como base el trabajo de Verplanken y Herabadi (2001), el cual comprende seis ítems. Por último, para la urgencia a comprar impulsivamente (UC) se tomó del trabajo de Liu et al. (2013) y comprende tres ítems.

El tiempo promedio de respuesta fue de 15 minutos por participante. Se logró una muestra de participación de 403 consumidores en Colombia. La mayoría de los participantes del estudio fueron compradores de entre 26 y 39 años - $55 \%$-; mujeres - $53 \%$-; en estado civil casado/unión — $52 \%$-; viven en la ciudad de Bogotá — $57 \%$-; tienen un nivel socioeconómico medio - $53 \%$-; y son empleados - $63 \%$ -

Los datos recopilados se analizaron con los programas SPSS y EQS 6.3, con el fin de observar la fiabilidad y validez del instrumento de medida, así como para contrastar las hipótesis propuestas en el trabajo.

\section{Resultados}

Tos datos recogidos en la investigación se analizaron en dos fases. La primera consiste en un análisis factorial confirmatorio (CFA) para verificar la fiabilidad y la validez del instrumento de medida. En la segunda fase se contrastan las hipótesis, primero de manera exploratoria a través de un análisis de correlaciones entre factores, y luego se ejecuta un análisis de ecuaciones estructurales con la finalidad de obtener resultados concluyentes.

\subsection{Fiabilidad y validez del instrumento de medida}

Para hallar la fiabilidad y la validez del instrumento de medida, se llevó a cabo un CFA con el software EQS 6.3. Los resultados son presentados en la tabla 1.

Tabla 1. Fiabilidad y validez del instrumento de medida

\begin{tabular}{|c|c|c|c|c|c|c|}
\hline Factor & I & Ítem & $\beta$ & $a \mathrm{de} \mathrm{Cr}$ & CR & AVE \\
\hline \multirow{3}{*}{$\begin{array}{l}\text { Disponibilidad } \\
\text { del producto }\end{array}$} & DP1 & $\begin{array}{l}\text { En esta tienda en línea encuentro variedad y disponibilidad } \\
\text { suficiente de productos. }\end{array}$ & 0,887 & \multirow{3}{*}{0,939} & \multirow{3}{*}{0,940} & \multirow{3}{*}{0,839} \\
\hline & DP2 & $\begin{array}{l}\text { En esta tienda en línea puedo encontrar con facilidad los } \\
\text { productos que necesito. }\end{array}$ & 0,933 & & & \\
\hline & DP3 & $\begin{array}{l}\text { En esta tienda en línea puedo encontrar con facilidad los } \\
\text { productos en los que estoy interesado/a. }\end{array}$ & 0,927 & & & \\
\hline \multirow{3}{*}{ Atractivo visual } & AV1 & La tienda en línea es visualmente agradable. & 0,926 & \multirow{3}{*}{0,957} & \multirow{3}{*}{0,958} & \multirow{3}{*}{0,885} \\
\hline & AV2 & La tienda en línea exhibe un diseño visualmente agradable. & 0,970 & & & \\
\hline & AV3 & El diseño de la tienda en línea es atractivo. & 0,925 & & & \\
\hline \multirow{4}{*}{ Telepresencia } & TP2 & $\begin{array}{l}\text { El mundo generado por el computador me parece más un } \\
\text { lugar que he visitado, que algo que he visto. }\end{array}$ & 0,649 & \multirow{4}{*}{0,879} & \multirow{4}{*}{0,881} & \multirow{4}{*}{0,653} \\
\hline & TP3 & $\begin{array}{l}\text { Cuando navego en la tienda en línea, me siento más en el } \\
\text { mundo del computador que en el mundo que me rodea. }\end{array}$ & 0,760 & & & \\
\hline & TP4 & $\begin{array}{l}\text { Me olvido del mundo a mi alrededor cuando estoy navegando } \\
\text { en la tienda en línea. }\end{array}$ & 0,908 & & & \\
\hline & TP5 & $\begin{array}{l}\text { Cuando salgo de la tienda en línea siento que vuelvo al mundo } \\
\text { real. }\end{array}$ & 0,887 & & & \\
\hline
\end{tabular}




\begin{tabular}{|c|c|c|c|c|c|c|}
\hline \multirow{3}{*}{$\begin{array}{l}\text { Reacción } \\
\text { cognitiva }\end{array}$} & $\mathrm{RC} 2$ & $\begin{array}{l}\text { Generalmente solo compro los productos que tenía planeado } \\
\text { comprar. }\end{array}$ & 0,741 & \multirow{3}{*}{0,802} & \multirow{3}{*}{0,805} & \multirow{3}{*}{0,580} \\
\hline & $\mathrm{RC} 4$ & $\begin{array}{l}\text { Solo compro cosas que realmente necesito en esta tienda en } \\
\text { línea. }\end{array}$ & 0,835 & & & \\
\hline & RC6 & $\begin{array}{l}\text { Antes de comprar algo en esta tienda en línea, siempre } \\
\text { considero cuidadosamente si lo necesito o no. }\end{array}$ & 0,702 & & & \\
\hline \multirow{3}{*}{$\begin{array}{l}\text { Urgencia a } \\
\text { comprar }\end{array}$} & $\mathrm{UC} 1$ & $\begin{array}{l}\text { Cuando navego en esta tienda en línea siento una urgencia de } \\
\text { comprar cosas distintas o adicionales a mi objetivo. }\end{array}$ & 0,746 & \multirow{3}{*}{0,879} & \multirow{3}{*}{0,884} & \multirow{3}{*}{0,720} \\
\hline & UC2 & $\begin{array}{l}\text { Navegando en la tienda en línea siento deseos de comprar } \\
\text { productos que no pertenecen a mi objetivo de compra } \\
\text { específico. }\end{array}$ & 0,892 & & & \\
\hline & UC3 & $\begin{array}{l}\text { Mientras navego en la tienda en línea tengo la inclinación a } \\
\text { comprar productos fuera de mi objetivo de compra específico. }\end{array}$ & 0,898 & & & \\
\hline
\end{tabular}

Nota. $\mathrm{X} 2=221,905$ (94df); NFI = 0,955; NNFI = 0,966; CFI = 0,973; SRMR = 0,051; RMSEA = 0,058; $\alpha$ de Cr = alfa de Cronbach, $\mathrm{CR}=$ fiabilidad compuesta, AVE = varianza extraída promedio.

Fuente. Elaboración propia.

De acuerdo con los resultados del análisis factorial exploratorio previo, se eliminaron los ítems TP1 de la escala de presencia social y RC1, RC3 y RC5 de la escala de estado cognitivo, con lo cual se mejora considerablemente los valores de la bondad de ajuste. Los resultados del CFA muestran que los valores del coeficiente alfa de Cronbach $-\alpha$ de $\mathrm{Cr}-$, aunque no tienen una regla que indique a partir de qué valor existe fiabilidad del instrumento, se toman de manera general menor a 0,25 como baja confiabilidad, 0,50 como fiabilidad media o regular, mayor a 0,75 como aceptable y mayor a 0,90 como elevada (Hernández, Fernández y Baptista, 2014).
Para la investigación, los valores de AV y DP determinan una confiabilidad elevada, mientras que el RC, TP y UC tienen valores que indican una confiabilidad aceptable. Lo anterior indica que existe una unidimensionalidad entre las variables $y$ las escalas de medición utilizadas. Igualmente, los valores de la fiabilidad compuesta de las escalas (CR) y la varianza media extraída (AVE) tienen buenos niveles, $>0,7$ y $>0,5$, respectivamente, de acuerdo con Bagozzi y Yi (1988). Comprobada la fiabilidad y la varianza extraída promedio de las escalas, el siguiente paso es comprobar la validez discriminante del instrumento de medida. Para esto se presenta el criterio Fornell y Larcker (1981) en la tabla 2.

Tabla 2. Validez discriminante criterio Fornell-Larcker

\begin{tabular}{|c|c|c|c|c|c|}
\hline & DP & AV & PS & RC & UC \\
\hline DP & $\mathbf{0 , 9 1 6}$ & & & & \\
\hline AV & 0,619 & $\mathbf{0 , 9 4 1}$ & & & \\
\hline TP & 0,319 & 0,279 & $\mathbf{0 , 8 0 8}$ & & \\
\hline RC & 0,128 & 0,112 & 0,031 & $\mathbf{0 , 7 6 1}$ & \\
\hline UC & 0,107 & 0,131 & 0,313 & $-0,220$ & $\mathbf{0 , 8 4 8}$ \\
\hline
\end{tabular}

Nota. La diagonal indica la raíz cuadrada del AVE (validez discriminante). Los datos en el triángulo inferior corresponden a las correlaciones entre los factores.

Fuente. Elaboración propia. 
De acuerdo con el criterio de Fornell y Larcker (1981), los valores en la diagonal deben ser superiores a los valores en el triángulo inferior para que se pueda determinar la validez discriminante. Según los resultados, los valores son apropiados y se puede confirmar la validez.

\subsection{Contrastación de las hipótesis}

El procesamiento de datos para contrastar las hipótesis planteadas en la investigación consiste en dos pasos. El primero, exploratorio, a fin de conocer la correlación entre los factores; el segundo, una comprobación a través de ecuaciones estructurales utilizando el software EQS 6.3. El resultado de las correlaciones bivariadas se realiza a través de una prueba de significación bilateral con coeficiente de Pearson; los resultados se presentan en la tabla 3.

Tabla 3. Correlaciones bivariadas entre los factores

\begin{tabular}{|c|c|c|c|c|c|c|}
\hline & & DP & AV & PS & RC & UC \\
\hline \multirow{2}{*}{ DP } & Correlación de Pearson & 1 & $0,602^{* *}$ & $0,314^{* *}$ & $0,107^{*}$ & $0,114^{*}$ \\
\hline & Sig. & & 0,000 & 0,000 & 0,032 & 0,023 \\
\hline \multirow{2}{*}{$\mathbf{A V}$} & Correlación de Pearson & & 1 & $0,296^{* *}$ & $0,103^{*}$ & $0,145^{* *}$ \\
\hline & Sig. & & & 0,000 & 0,040 & 0,004 \\
\hline \multirow{2}{*}{$\mathbf{T P}$} & Correlación de Pearson & & & 1 & 0,042 & $0,299^{* *}$ \\
\hline & Sig. & & & & 0,405 & 0,000 \\
\hline \multirow{2}{*}{$\mathbf{R C}$} & Correlación de Pearson & & & & 1 & $-0,182^{* *}$ \\
\hline & Sig. & & & & & 0,000 \\
\hline
\end{tabular}

${ }^{* *} \mathrm{p}<0,01 ;{ }^{*} \mathrm{p}<\mathrm{M} 0,05$

Fuente. Elaboración propia.

De acuerdo con el resultado preliminar de las correlaciones entre factores, se evidencia una posible relación entre la disponibilidad del producto y la telepresencia $-\mathrm{H} 1-$ y la respuesta cognitiva $-\mathrm{H} 2-$. En cuanto al atractivo social, podría ser un antecedente importante de la telepresencia $-\mathrm{H} 3-$ y de la respuesta cognitiva - H4 - La urgencia por comprar por su parte podría ser explicada a través de la telepresencia - H5 - y la reacción cognitiva $-\mathrm{H} 6-$, siendo esta relación negativa.

Si bien las correlaciones dan una idea general de la interacción entre los factores, las ecuaciones estructurales determinan la dirección y el tipo de relación entre estos de manera concluyente; así, se presenta en la tabla 4 el resultado del análisis del modelo de ecuaciones estructurales SEM con el software EQS 6.3.

Tabla 4. Ecuaciones estructurales

\begin{tabular}{|c|c|c|c|c|}
\hline H & Hipótesis & Resultado & $\boldsymbol{\beta}$ & t \\
\hline H1 & Disponibilidad percibida del producto $\rightarrow$ reacción cognitiva & Rechazada & 0,095 & 1,285 \\
\hline H2 & Disponibilidad percibida del producto $\rightarrow$ telepresencia & Aceptada & $0,237^{*}$ & 3,460 \\
\hline H3 & Atractivo visual $\rightarrow$ reacción cognitiva & Rechazada & 0,050 & 0,686 \\
\hline H4 & Atractivo visual $\rightarrow$ telepresencia & Aceptada & $0,135^{*}$ & 2,025 \\
\hline H5 & Reacción cognitiva $\rightarrow$ urgencia a comprar impulsivamente & Aceptada & $-0,229^{*}$ & 4,037 \\
\hline H6 & Telepresencia $\rightarrow$ urgencia a comprar impulsivamente & Aceptada & $0,323^{*}$ & 5,665 \\
\hline
\end{tabular}

Nota. X2(df) = 223,919(97) NFI = 0,954; NNFI = 0,967; CFI = 0,973; SRMR=0.054; RMSEA = 0,057; * $<<0,01$

Fuente. Elaboración propia. 
A partir del análisis de ecuaciones estructurales se observa en el modelo que las hipótesis $\mathrm{H} 1 \mathrm{y}$ $\mathrm{H} 3$ son rechazadas. Las relaciones contrastadas positivamente corresponden a: disponibilidad percibida del producto $\rightarrow$ telepresencia $\mathrm{H} 2-$, atractivo visual $\rightarrow$ telepresencia - $\mathrm{H} 4-$, telepresencia $\rightarrow$ urgencia a comprar impulsivamente - H6 - En la hipótesis 5 se confirma una relación directa y negativa — reacción cognitiva $\rightarrow$ Urgencia a comprar impulsivamente-.

\subsection{Discusión de los resultados}

Los resultados confirman del modelo que el atractivo visual y la disponibilidad percibida del producto en un sitio web de compras en grupo favorecen de forma positiva la telepresencia percibida por el usuario. De igual forma, la telepresencia percibida por el usuario favorecerá de forma positiva a la urgencia a comprar por impulso. Por otro lado, los mismos estímulos no determinan una relación con la variable del estado cognitivo, aunque sí se observa una relación del estado cognitivo con la urgencia a comprar por impulso; sin embargo, esta no favorece la tendencia a realizar compras por impulso.

La disponibilidad percibida del producto y el atractivo visual, con relación a la reacción cognitiva, se analizaron a través de las hipótesis H1 y H3. Los resultados señalan que no existe relación de estas variables con el estado cognitivo, lo cual se debe, para el caso de la disponibilidad percibida del producto, según Aydinli et al. (2017), a que la atracción de los consumidores hacia grandes surtidos responde a procesos afectivos más que a procesos cognitivos; además, para el caso del atractivo visual, el diseño del sitio web y la interactividad/personalización favorecen las gratificaciones hedónicas $y$, por tanto, conducen a impulsos de compra (Bressolles, Durrieu y Giraud, 2007).

De modo que los consumidores con características hedónicas serán más propensos a participar en la búsqueda de información exploratoria que en búsquedas utilitarias, lo que alienta más las compras por impulso (Kim y Eastin, 2011). Los compradores que planean sus compras a menudo buscan comparar los precios en diferentes tiendas en línea frente a buscar gangas, como en el caso de quienes no planean sus compras (Wolfinbarger y Gilly, 2001).

Por otro lado, de acuerdo con los resultados del estudio empírico, se encuentra que la reacción cognitiva incide de forma directa, pero negativa, en la urgencia de compra por impulso - hipótesis 5-. Una explicación surge porque el estado cognitivo se refiere al procesamiento y la interpretación de información que realiza el consumidor (Eroglu et al., 2001), así como el proceso de compra por impulso se caracteriza por ser espontáneo y decidirse sobre la marcha (Verhagen y van Dolen, 2011). Es decir, una sensación repentina que disminuye el procesamiento de información y las reacciones cognitivas (Floh y Madlberger, 2013).

Por tanto, la compra por impulso se relaciona más con una alta activación emocional y un bajo control cognitivo (Sharma, Sivakumaran y Marshall, 2010). Otra posible explicación se debe al hecho de que los productos o servicios ofrecidos en los sitios de compras en grupo en línea son productos que pueden evaluarse con facilidad antes de la compra, lo cual representa un bajo riesgo para el consumidor $y$, por tanto, brinda participación a una compra de procesamiento más afectiva que racional (Verhagen y Bloemers, 2018).

De igual forma, los resultados muestran que, en el proceso de compras por impulso, el consumidor debe percibir este comportamiento como razonable o adecuado (Chen y Yao, 2018). Sin embargo, los participantes mostraron una inclinación a no considerar evaluaciones durante la compra, con lo que se demuestra que las evaluaciones racionales sobre las compras disminuirán el impulso de compra.

De acuerdo con la relación entre la disponibilidad percibida del producto y el atractivo visual frente a la telepresencia — hipótesis 2 y 4 -, empíricamente se confirman estos supuestos y se obtiene un coeficiente mayor en la relación entre la disponibilidad percibida del producto y la telepresencia. Esto se debe a que los compradores tienen la percepción de encontrar una mejor selección de productos en tiendas en línea, en general, con relación a las tiendas físicas. 
En consecuencia, una de las actividades del consumidor es buscar ofertas en línea (Wolfinbarger y Gilly, 2001) y una gran variedad de productos generará más sentimientos positivos (Yoo et al., 1998), de modo que la telepresencia funciona como un mediador entre la actitud del consumidor y su intención de compra, pues reduce la distancia entre el producto en línea y el real (Suh y Chang, 2006). Además, la experiencia que crea la telepresencia simula la observación el producto, aunque este no se encuentre físicamente y así logra persuadir al comprador (Kim y Biocca, 1997).

Con respecto al atractivo visual, aunque presenta un coeficiente menor, es aún una variable importante porque la telepresencia puede ser manipulada por los elementos de diseño de una interfaz web (Lim y Ayyagari, 2018), en vista de que las características visuales de la pantalla inducen al consumidor a una sensación de presencia por medio de la computadora, es decir, una experiencia similar de interactividad con los productos físicos y con los virtuales (Coyle y Thorson, 2001).
Finalmente, los resultados del análisis mostraron que la telepresencia incide de forma directa y positiva en la urgencia a comprar por impulso - hipótesis 6 - porque, como explican Lee y Park (2014), entre más nivel de telepresencia sienta el cliente en una tienda en línea, menos será la limitación espacial; así, menos información del producto o interacción con el entorno de la tienda llevará a menos influencia en la respuesta del consumidor.

De este modo, la telepresencia podría satisfacer las necesidades de los consumidores, así como afectar sus actitudes y comportamientos (Gao et al., 2018), además de que el atractivo visual y la mercancía serán estímulos importantes en los entornos de compra por impulso; en este sentido, se requieren los ambientes tranquilos, amistosos, bien informados, divertidos y con un surtido atractivo (Verhagen y van Dolen, 2011).

\section{Conclusiones, limitaciones y futuras líneas de investigación}

$\mathrm{L}$ a investigación realizada ha permitido conocer Jel impacto positivo que tiene la telepresencia en la urgencia por comprar impulsivamente a través de canales en línea. A la vez, ha demostrado que un estado cognitivo superior tiene un efecto negativo en esta urgencia. El raciocinio del consumidor en los procesos de decisión de compra es importante a fin de lograr relaciones comerciales a largo plazo, pues un consumidor que perciba que una tienda ha usado las emociones para generar una compra tendrá una disonancia cognitiva en el periodo poscompra, lo cual lo llevará a sentimientos de insatisfacción y frustración.

La urgencia para comprar impulsivamente pueden gestionarla las tiendas en ciertos momentos cuando se requiere realizar una actividad promocional, bien sea para dar a conocer la empresa o bien para salir de algún stock, de modo que se realiza un seguimiento posventa con miras a cuidar la relación comercial incipiente. No puede gestionarse como una actividad promocional regular en una empresa que espere durar en el mercado.

Este estudio logra abarcar resultados que se pueden generalizar dada la muestra poblacional. Sin embargo, se encuentra susceptible a limitaciones. En primer lugar, aunque en primera instancia se habían propuesto dos variables mediadoras - telepresencia como parte de la reacción afectiva y el estado cognitivo como reacción cognitiva-, los resultados no arrojaron valores consistentes al realizar la relación entre los estímulos y la reacción cognitiva. Por esta razón, los autores proponen incluir variables moderadoras como aspectos demográficos de los participantes —edad, ocupación, estrato, etc.-, 
un aspecto que podría arrojar resultados distintos. En segundo lugar, al evaluar la reacción afectiva se consideró una sola variable mediadora; por lo tanto, en futuras investigaciones sería importante considerar otro conjunto de emociones que permitan identificar el papel del organismo en la compra por impulso en línea.

\section{Referencias}

Applebaum, W. (1951). Studying customer behavior in retail stores. Journal of Marketing, 16(2), 172-178. DOI: https:// doi.org/10.1177/002224295101600204

Aydinli, A.; Gu, Y.; Pham, M. T. (2017). An experienceutility explanation of the preference for larger assortments. International Journal of Research in Marketing, 34(3), 746-760. DOI: https://doi.org/10.1016/j.ijresmar.2017.06.007

Bagozzi, R. P.; Yi, Y. (1988). On the evaluation of structural equation models. Journal of Academy Marketing Sciences, 16(1), 74-94. DOI: https://doi.org/10.1007/BF02723327

Beatty, S. E.; Ferrell, M. E. (1998). Impulse buying: modeling its precursors. Journal of Retailing, 74(2), 169-191. DOI: https:// doi.org/10.1016/S0022-4359(99)80092-X

Bitner, M. J. (1992). Servicescapes: the impact of physical surroundings on customers and employees. Journal of Marketing, 56(2), 57-71. DOI: https://doi. org/10.1177/002224299205600205

Boon, E.; Pitt, L.; Ofek, N. (2015). «Deal of the day»: an analysis of subscriber purchase behavior. Tourism and Hospitality Research, 15(2), 105-114. DOI: https://doi. org/10.1177/1467358414567798

Bressolles, G.; Durrieu, F.; Giraud, M. (2007). The impact of electronicservice quality's dimensions on customer satisfaction and buying impulse. Journal of Customer Behaviour, 6(1), 3756. DOI: https://doi.org/10.1362/147539207X198365

Burroughs, J. (1996). Product symbolism, self-meaning, and holistic matching: the role of information processing in impulsive buying. Advances in Consumer Research, 23, 463469. Recuperado de https://bit.ly/32DWuC9

Chan, T. K.; Cheung, C. M.; Lee, Z. W. (2016). The state of online impulse-buying research: a literature analysis. Information \& Management, 54(2), 204-217. DOI: https:// doi.org/10.1016/j.im.2016.06.001

Chang, S. H.; Chih, W. H.; Liou, D. K.; Hwang, L. R. (2014). The influence of web aesthetics on customers' PAD. Computers in Human Behavior, 36, 168-178. DOI: https://doi. org/10.1016/j.chb.2014.03.050
Chen, C. C.; Yao, J. Y. (2018). What drives impulse buying behaviors in a mobile auction? The perspective of the StimulusOrganism-Response model. Telematics and Informatics, 35(5), 1249-1262.DOI: https://doi.org/10.1016/j.tele.2018.02.007

Chen, J.; Kauffman, R. J.; Liu, Y.; Song, X. (2010). Segmenting uncertain demand in group-buying auctions. Electronic Commerce Research and Applications, 9(2), 126-147. DOI: https://doi.org/10.1016/j.elerap.2009.03.001

Coley, A.; Burgess, B. (2003). Gender differences in cognitive and affective impulse buying. Journal of Fashion Marketing and Management: An International Journal, 7(3), 282-295. DOI: https://doi.org/10.1108/13612020310484834

Coyle, J. R.; Thorson, E. (2001). The effects of progressive levels of interactivity and vividness in web marketing sites. Journal of Advertising, 30(3), 65-77. DOI: https://doi.org/10.1080/009 13367.2001.10673646

Erdogmus, I. E.; Cicek, M. (2011). Online group buying: what is there for the consumers. Procedia Social and Behavioral Sciences, 24, 308-316. DOI: https://doi.org/10.1016/j. sbspro.2011.09.138

Eroglu, S. A.; Machleit, K. A.; Davis, L. M. (2001). Atmospheric qualities of online retailing: a conceptual model and implications. Journal of Business research, 54(2), 177-184. DOI: https://doi.org/10.1016/S0148-2963(99)00087-9

Flapper, S. D. P.; González-Velarde, J. L.; Smith, N. R.; EscobarSaldívar, L. J. (2010). On the optimal product assortment: comparing product and customer-based strategies. International Journal of Production Economics, 125(1), 167-172. DOI: https://doi.org/10.1016/j.ijpe.2010.01.017

Floh, A.; Madlberger, M. (2013). The role of atmospheric cues in online impulse-buying behavior. Electronic Commerce Research and Applications, 12(6), 425-439. DOI: https://doi. org/10.1016/j.elerap.2013.06.001

Fornell, C.; Larcker, D. F. (1981). Structural equation models with unobservable variables and measurement error: algebra and statistics. Journal of Marketing Research, 18(3), 382-388. DOI: https://doi.org/10.1177/002224378101800313

Gao, W.; Liu, Y.; Liu, Z.; Li, J. (2018). How does presence influence purchase intention in online shopping markets? An explanation based on self-determination theory. Behaviour \& Information Technology, 37(8), 786-799. DOI: https://doi. org/10.1080/0144929X.2018.1484514

Gillenson, M. L.; Sherrell, D. L. (2002). Enticing online consumers: an extended technology acceptance perspective. Information \& Management, 39(8), 705-719. DOI: https:// doi.org/10.1016/S0378-7206(01)00127-6

Hassanein, K.; Head, M. (2005). The impact of infusing social presence in the web interface: an investigation across product types. International Journal of Electronic Commerce, 10(2), 3155. DOI: https://doi.org/10.2753/JEC1086-4415100202 
He, Y.; Oppewal, H. (2018). See how much we've sold already! effects of displaying sales and stock level information on consumers' online product choices. Journal of Retailing, 94(1), 45-57. DOI: https://doi.org/10.1016/j.jretai.2017.10.002

Heeter, C. (2003). Reflections on real presence by a virtual person. Presence. Teleoperators \& Virtual Environments, 12(4), 335345. DOI: https://doi.org/10.1162/105474603322391587

Hernández, R.; Fernández, C.; Baptista, P. (2014). Metodología de la investigación. Ciudad de México: McGraw-Hill.

Iglesias, I. (01 de abril de 2015). Groupon Colombia ofrecerá nueva web y más servicios. Portafolio. Recuperado de https:// bit.ly/3gtZ0TB

Jang, S. S.; Namkung, Y. (2009). Perceived quality, emotions, and behavioral intentions: application of an extended Mehrabian-Russell model to restaurants. Journal of Business Research, 62(4), 451-460. DOI: https://doi.org/10.1016/j. jbusres.2008.01.038

Kauffman, R. J.; Wang, B. (2001). New buyers' arrival under dynamic pricing market microstructure: the case of groupbuying discounts on the Internet. Journal of Management Information Systems, 18(2), 157-188. DOI: https://doi.org/1 $0.1080 / 07421222.2001 .11045687$

Kauffman, R. J.; Wang, B. (2002). Bid together, buy together: on the efficacy of group-buying business models in Internetbased selling. En P.B. Lowry; J.O. Cherrington; R.R Watson (Eds.) Handbook of Electronic Commerce in Business and Society. (99-137). Boca Raton: CRC Press.

Kim, S.; Eastin, M. S. (2011). Hedonic tendencies and the online consumer: an investigation of the online shopping process. Journal of Internet Commerce,10(1), 68-90. DOI: https://doi. org/10.1080/15332861.2011.558458

Kim, T.; Biocca, F. (1997). Telepresence via television: two dimensions of telepresence may have different connections to memory and persuasion. Journal of Computer-Mediated Communication, 3(2), JCMC325. DOI: https://doi. org/10.1111/j.1083-6101.1997.tb00073.x

Klaus, P.; Nguyen, B. (2013). Exploring the role of the online customer experience in firms' multi-channel strategy: an empirical analysis of the retail banking services sector. Journal of Strategic Marketing, 21(5), 429-442. DOI: https://doi. org/10.1080/0965254X.2013.801610

Koufaris, M.; Kambil, A.; LaBarbera, P. (2001). Consumer behavior in web-based commerce: an empirical study. International Journal of Electronic Commerce, 6(2), 115-138. DOI: https://doi.org/10.1080/10864415.2001.11044233

Langviniene, N.; Zemblyte, J.; Sliziene, G. (2016). What influences buying leisure services coupons on online group buying? Engineering Economics, 27(3), 345-356. DOI: https:// doi.org/10.5755/j01.ee.27.3.12944
Lee, E. J.; Park, J. (2014). Enhancing virtual presence in e-tail: dynamics of cue multiplicity. International Journal of Electronic Commerce, 18(4), 117-146. DOI: https://doi.org/10.2753/ JEC1086-4415180405

Lim, J.; Ayyagari, R. (2018). Investigating the determinants of telepresence in the e-commerce setting. Computers in Human Behavior, 85, 360-371. DOI: https://doi.org/10.1016/j. chb.2018.04.024

Lim, Y. M.; Cham, T. H. (2015). A profile of the Internet shoppers: evidence from nine countries. Telematics and Informatics, 32(2), 344-354. DOI: https://doi.org/10.1016/j. tele.2014.10.002

Lindgaard, G.; Dudek, C.; Sen, D.; Sumegi, L.; Noonan, P. (2011). An exploration of relations between visual appeal, trustworthiness and perceived usability of homepages. ACM Transactions on Computer-Human Interaction (TOCHI), 18(1), 30. DOI: https://doi.org/10.1145/1959022.1959023

Lins, S.; Dóka, Á.; Bottequin, E.; Odabašić, A.; Pavlović, S.; Merchán, A.; Golasa, A.; Hylander, F. (2015). The effects of having, feeling, and thinking on impulse buying in European adolescents. Journal of International Consumer Marketing, 27(5), 414-428. DOI: https://doi.org/10.1080/08961530.2 015.1027028

Liu, Y.; Li, H.; Hu, F. (2013). Website attributes in urging online impulse purchase: an empirical investigation on consumer perceptions. Decision Support Systems, 55(3), 829-837. DOI: https://doi.org/10.1016/j.dss.2013.04.001

López, G. B. (2006). Una aproximación a la psicología ambiental. Fundamentos en Humanidades, (13), 157-168.

Madhavaram, S. R.; Laverie, D. A. (2004). Exploring impulse purchasing on the Internet. NA-Advances in Consumer Research, 31, 59-66. Recuperado de https://bit.ly/3sJ3HeI

Mehrabian, A.; Russell, J. A. (1974). An approach to environmental psychology. Cambridge: MIT Press.

Morales, A.; Kahn, B. E.; McAlister, L.; Broniarczyk, S. M. (2005). Perceptions of assortment variety: the effects of congruency between consumers' internal and retailers' external organization. Journal of Retailing, 81(2), 159-169. DOI: https://doi.org/10.1016/j.jretai.2005.03.007

Mummalaneni, V. (2005). An empirical investigation of web site characteristics, consumer emotional states and on-line shopping behaviors. Journal of Business Research, 58(4), 526532. DOI: https://doi.org/10.1016/S0148-2963(03)001437

Nakhata, C.; Kuo, H. C. (2017). Consumer avoidance of specially priced items during social coupon redemption. Journal of Retailing and Consumer Services, 34, 287-293. DOI: https:// doi.org/10.1016/j.jretconser.2016.01.011 
Ni, G.; Xu, Y.; Xu, J.; Dong, Y. (2015). Optimal decisions for fixed-price group-buying business originated in China: a game theoretic perspective. International Journal of Production Research, 53(10), 2995-3005. DOI: https://doi.org/10.1080 /00207543.2014.965350

Ning-Shen, K.; Khalifa, M. (2012). System design effects on online impulse buying. Internet Research, 22(4), 396-425. DOI: https://doi.org/10.1108/10662241211250962

Oh, J.; Fiorito, S. S.; Cho, H.; Hofacker, C. F. (2008). Effects of design factors on store image and expectation of merchandise quality in web-based stores. Journal of Retailing and Consumer Services, 15(4), 237-249. DOI: https://doi.org/10.1016/j. jretconser.2007.03.004

Ou, C. X.; Pavlou, P.; Davison, R. (2013). Swift guanxi in online marketplaces: the role of computer-mediated communication technologies. MIS Quarterly, 38(1), 209-230. DOI: https:// doi.org/10.25300/MISQ/2014/38.1.10

Parboteeah, D. V.; Valacich, J. S.; Wells, J. D. (2009). The influence of website characteristics on a consumer's urge to buy impulsively. Information Systems Research, 20(1), 60-78. DOI: https://doi.org/10.1287/isre.1070.0157

Piron, F. (1991). Defining impulse purchasing. NA-Advances in Consumer Research, 18, 509-514. Recuperado de http:// acrwebsite.org/volumes/7206/volumes/v18/NA-18

Richards, T. J.; Hamilton, S. F.; Allender, W. (2016). Search and price dispersion in online grocery markets. International Journal of Industrial Organization, 47, 255-281. DOI: https:// doi.org/10.1016/j.ijindorg.2016.05.004

Rook, D. W. (1987). The buying impulse. Journal of Consumer Research, 14(2), 189-199. DOI: https://doi. org/10.1086/209105

Rook, D. W.; Gardner, M. P. (1993). In the mood: Impulse buying's affective antecedents. Research in Consumer Behavior, 6(7), 1-28. Recuperado de https://bit.ly/3xjq6mo

Sharma, P.; Sivakumaran, B.; Marshall, R. (2010). Impulse buying and variety seeking: a trait-correlates perspective. Journal of Business Research, 63(3), 276-283. DOI: https://doi. org/10.1016/j.jbusres.2009.03.013

Skulmowski, A.; Augustin, Y.; Pradel, S.; Nebel, S.; Schneider, S.; Rey, G.D. (2016). The negative impact of saturation on website trustworthiness and appeal: a temporal model of aesthetic website perception. Computers in Human Behavior, 61, 386393. DOI: https://doi.org/10.1016/j.chb.2016.03.054

Sofi, S. A.; Najar, S. A. (2018). Impact of personality influencers on psychological paradigms: an empirical-discourse of big five framework and impulsive buying behaviour. European Research on Management and Business Economics, 24(2), 71-81. DOI: https://doi.org/10.1016/j.iedeen.2017.12.002
Song, H. G.; Chung, N.; Koo, C. (2015). Impulsive buying behavior of restaurant products in social commerce: a role of serendipity and scarcity message. Ponencia presentada en PACIS 2015: Pacific Asia Conference on Information Systems. Singapur, 5-9 de julio. Recuperado de https://bit.ly/2RS1JMp

Steinhart, Y.; Mazursky, D.; Kamins, M. A. (2013). The process by which product availability triggers purchase. Marketing Letters, 24(3), 217-228. DOI: https://doi.org/10.1007/s11002-0139227-4

Stern, H. (1962). The significance of impulse buying today. The Journal of Marketing, 26(2), 59-62. DOI: https://doi. org/10.1177/002224296202600212

Steuer, J. (1992). Defining virtual reality: dimensions determining telepresence. Journal of Communication, 42(4), 73-93. DOI: https://doi.org/10.1111/j.1460-2466.1992.tb00812.x

Suh, K. S.; Chang, S. (2006). User interfaces and consumer perceptions of online stores: the role of telepresence. Behaviour \& Information Technology, 25(2), 99-113. DOI: https://doi. org/10.1080/01449290500330398

Verhagen, T.; Bloemers, D. (2018). Exploring the cognitive and affective bases of online purchase intentions: a hierarchical test across product types. Electronic Commerce Research, 18(3), 537-561. DOI: https://doi.org/10.1007/s10660-017-9270-y

Verhagen, T.; van Dolen, W. (2011). The influence of online store beliefs on consumer online impulse buying: a model and empirical application. Information \& Management, 48(8), 320327. DOI: https://doi.org/10.1016/j.im.2011.08.001

Verplanken, B.; Herabadi, A. (2001). Individual differences in impulse buying tendency: feeling and no thinking. European Journal of Personality, 15(S1), S71-S83. DOI: https://doi. org/10.1002/per.423

Vieira, V. A. (2013). Stimuli-organism-response framework: a meta-analytic review in the store environment. Journal of Business Research, 66(9), 1420-1426. DOI: https://doi. org/10.1016/j.jbusres.2012.05.009

Vonkeman, C.; Verhagen, T.; van Dolen, W. (2017). Role of local presence in online impulse buying. Information \& Management, 54(8), 1038-1048. DOI: https://doi. org/10.1016/j.im.2017.02.008

Wang, J. J.; Zhao, X.; Li, J. J. (2013). Group buying: a strategic form of consumer collective. Journal of Retailing, 89(3), 338351. DOI: https://doi.org/10.1016/j.jretai.2013.03.001

Wang, L. C.; Baker, J.; Wagner, J. A.; Wakefield, K. (2007). Can a retail web site be social? Journal of Marketing, 71(3), 143-157. DOI: https://doi.org/10.1509/jmkg.71.3.143

Wolfinbarger, M.; Gilly, M. C. (2001). Shopping online for freedom, control, and fun. California Management Review, 43(2), 34-55. DOI: https://doi.org/10.2307/41166074 
Xiang, L.; Zheng, X.; Lee, M. K.; Zhao, D. (2016). Exploring consumers' impulse buying behavior on social commerce platform: the role of parasocial interaction. International Journal of Information Management, 36(3), 333-347. DOI: https://doi.org/10.1016/j.ijinfomgt.2015.11.002

Xiao, S. H.; Nicholson, M. (2013). A multidisciplinary cognitive behavioural framework of impulse buying: a systematic review of the literature. International Journal of Management Reviews, 15(3), 333-356. DOI: https://doi.org/10.1111/j.1468 2370.2012.00345. $\mathrm{x}$

Yoo, C.; Park, J.; MacInnis, D. J. (1998). Effects of store characteristics and in-store emotional experiences on store attitude. Journal of Business Research, 42(3), 253-263. DOI: https://doi.org/10.1016/S0148-2963(97)00122-7

Zhang, G.; Shang, J.; Yildirim, P. (2016). Optimal pricing for group buying with network effects. Omega, 63, 69-82. DOI: https://doi.org/10.1016/j.omega.2015.10.003

Zheng, X.; Men, J.; Yang, F.; Gong, X. (2019). Understanding impulse buying in mobile commerce: an investigation into hedonic and utilitarian browsing. International Journal of Information Management, 48, 151-160. DOI: https://doi. org/10.1016/j.ijinfomgt.2019.02.010

Zhou, G.; Xu, K.; Liao, S. S. (2013). Do starting and ending effects in fixed-price group-buying differ? Electronic Commerce Research and Applications, 12(2), 78-89. DOI: https://doi. org/10.1016/j.elerap.2012.11.006 